\title{
La revisión del servicio público audiovisual británico tras el informe de Lord Hutton
}

\author{
Juan Luis Manfredi Sánchez \\ Decano de la Facultad de Ciencias Humanas, Sociales y de la Comunicación. \\ Universidad SEK \\ Correo electrónico: jljucar@yahoo.es
}

\section{Resumen}

El caso Kelly ha fortalecido a la BBC. Los fallos en los procesos de producción y control de calidad de los servicios informativos han derivado hacia una crisis de proporciones gigantescas. En medio de la crisis periodística mundial, la Corporación ha sabido reponerse y regenerar los servicios informativos. El informe Neil, redactado tras la aparición del informe de Lord Hutton, revisa los criterios de producción periodística y elabora un catálogo de pautas para la elaboración de noticias con la finalidad de recuperar la credibilidad.

\begin{abstract}
The BBC has became stronger due to the Kelly affaire. The failure of the journalistic production process and the lack of quality controls instigated the biggest crisis of confidence in the Corporation's history. However, it has regenerate its own. The Neil Report, after the Lord Hutton Inquiry, checks the production criteria and elaborates an summary of guides in order to recover its credibility.
\end{abstract}

Palabras clave: televisión pública, BBC, veracidad, Iraq.

Key words: public service television, $B B C$, truthfulness, Iraq 


\section{EL CASO KELLY Y EL INFORME DE LORD HUTTON}

\subsection{Cronología y hechos}

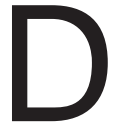

urante los meses de abril a junio de 2002, el ministerio de Defensa consulta a David Kelly, un experto en armamento en relación con las armas de destrucción masiva del ejército iraquí. El 24 de septiembre de 2002, Tony Blair, primer ministro laborista, hace público un informe sobre las armas de destrucción masiva del régimen iraquí de Saddam Hussein, así como la capacidad de éste de tener disponibles bombas químicas en 45 minutos. Tal información aparecía recogida en una serie de documentos del Ministerio de Defensa, que circularon durante el mes de septiembre en sucesivos borradores. En palabras de Alastair Campbell, director de Comunicaciones y Estrategia del gobierno, el informe principal necesitaba ser sustancialmente retocado antes de ser hecho público. Durante el último trimestre del año, se descubre que el citado informe incluye muchas informaciones incorrectas o no exactamente válidas. El escándalo salta cuando se descubren páginas y páginas de una tesis doctoral defendida hacía doce años.

En febrero de 2003, Colin Powell, secretario de Estado de Estados Unidos presenta ante el Consejo de Seguridad de Naciones Unidas las pruebas contra el gobierno iraquí de Saddam Hussein. Poco después, Donald Rumsfeld manifiesta que las armas existen, pero que probablemente se han trasladado a un país vecino. El gobierno acusa a la BBC de ser "manifiestamente hostil" en todo lo referente a Iraq y de presentar una cobertura "poco patriótica". Por ejemplo, el doctor Kelly había declarado a una conocida periodista que el informe presentaba la realidad muy radicalizada ("in a very black and white way"). Quince días después se entrevista con Andrew Gilligan, redactor del programa "Today" de Radio 4's.

El 29 de mayo de 2003, Andrew Gilligan acusa durante el informativo matinal al gobierno británico de adornar ("sexed up") el citado informe sobre la capacidad iraquí de montar las bombas químicas en 45 minutos. En la misma pieza, Gilligan aseguraba que Alastair Campbell había presionado a los servicios secretos para que exageraran el peligro de los arsenales iraquíes. Ante las primeras quejas, Gilligan decide mantener su fuente en secreto, aunque revela que se trata de "una fuente de inteligencia", maquillando que era sólo un experto en armamento a sueldo del Ministerio de Defensa. Gilligan repite su argumentación en una columna del tabloide The Sun el domingo siguiente, añadiendo detalles sobre la información facilitada por su fuente confidencial.

La BBC se niega a revelar la fuente, a pesar de las constantes presiones gubernamentales. Al tiempo, comienza una "caza de brujas" en el Ministerio de Defensa, que es denunciada por Susan Watts, corresponsal política del informativo nocturno. Ella afirma que un "oficial senior" presionó a los servicios secretos para que insistieran en la idea de los 45 minutos.

El 6 de junio Alastair Campbell, subrepticiamente acusado por la BBC, entrega un comunicado en el que asegura que la información de Andrew Gilligan presenta 
una serie de incorrecciones. Tal acusación es contestada por el propio Gilligan desde la columna dominical del diario The Sun. A mediados de junio se reúne un alto comité de Asuntos Exteriores, con el objeto de aclarar la situación. Gilligan declara ante el comité que su fuente era un "senior official" que había colaborado en la elaboración del informe. Por el contrario, Campbell atestigua que la información de la BBC es "completa y totalmente incierta... de hecho es una mentira". Al día siguiente, escribe a la BBC y reclama una disculpa pública. Richard Sambrook, jefe de informativos de la cadena, asegura que está siendo presionado desde el gobierno a unos niveles sin precedentes.

En julio de 2003, David Kelly, científico titular del Ministerio de Defensa, confiesa haberse entrevistado con Andrew Gilligan, si bien asegura que él no es la fuente principal que ha informado al periodista. Acuerdan una declaración oficial manteniendo su nombre en secreto, aunque el 5 de julio The Times le señala como la fuente. El ministro de Defensa, Geoffrey Hoon, identifica públicamente a Kelly como el chivato y reclama por escrito a Gavyn Davies, presidente de la Corporación, para que revele si Kelly era realmente la fuente. La BBC ni confirma ni desmiente y muestra su total apoyo al periodista que redactó la información. El 10 de julio Kelly aparece señalado como fuente en The Times, The Guardian y The Financial Times. Ante el revuelo, Kelly es llamado a declarar ante la Comisión de Exteriores de la Cámara de los Comunes y reitera que él ha hablado con Gilligan, pero que ha declarado que el informe esté adornado ("sexep up"). El ministro mantiene que Kelly es el "topo", pero, junto a Tony Blair, reclama que la BBC también lo reconozca. Al tiempo, Tony Blair declara que aunque se equivocaran con la cuestión de las armas, se habría acabado con la amenaza del terrorismo.

El 17 de julio, el doctor Kelly sale a pasear a las tres de la tarde. Cuando a las doce de la noche no ha regresado, su familia llama a la policía. Al día siguiente es encontrado muerto, aparentemente a causa de un suicidio. Su familia asegura que sufría una fuerte presión personal y social. Los medios de comunicación reconocen que ha sido víctima de la lucha entre el Gobierno y la Corporación. Tony Blair vive sus horas más bajas. El 20 de julio, la BBC, de mutuo acuerdo con la familia del fallecido, confirma que Kelly era la fuente de las informaciones de Gilligan y Susan Watts.

El 21 de julio se crea una comisión para investigar las causas de la muerte de Kelly, presidida por el juez Hutton. Ese mismo día, el editor de Mail on Sunday acusa a Campbell de estar fuera de control y que su estado es peligroso, por claros desequilibrios mentales. Se entrevista con todos los implicados y se hacen públicos los documentos oficiales que ponen en tela de juicio las razones para la invasión de Iraq. Durante el proceso, se deja al descubierto la manipulación del gobierno en los informes sobre Iraq, algo que siempre se negó. Blair, que también fue llamado a declarar, asegura ante Lord Hutton que los informes son veraces, porque si fueran falsos hubiera dimitido. El 29 de agosto, Campbell dimite de su cargo, asegurando que el informe de Lord Hutton no tiene nada que ver con su decisión. 
Un elemento interesante a considerar en la valoración del caso es la relación establecida entre el gobierno laborista y el grupo de comunicación News Corporation, presidido por Rupert Murdoch. Algunos autores señalan que el apoyo de la British Sky Broadcasting (BSkyB), líder de la televisión de pago, Fox, The Sun y The Times a la guerra de Iraq y a las campañas de información tendrán como recompensa una licencia de televisión digital terrestre en abierto ${ }^{1}$. Hay que recordar la información en exclusiva sobre el caso publicada por The Sun y que todos los medios estimaban como filtrada directamente desde el gabinete de Alastair Campbell.

Frente a la crisis abierta, la BBC comenzaba el año 2004 como líder de audiencia, pero con problemas de credibilidad.

\subsection{Las conclusiones del informe de Lord Hutton}

El 29 de enero se hace público el informe, que ya había sido entregado a los líderes de la oposición y que The Sun, del grupo de Murdoch, adelantó en horas. Poco después el propio Hutton aseguró que investigaría esta filtración. Según el informe, David Kelly es el único responsable de su suicidio, ya que cometió el error de hablar con la BBC. Al darse cuenta de su error, la presión le condujo al suicidio. En cualquier caso, estima Hutton, el experto se expuso al riesgo de tales presiones al pasar de manera indebida información a la prensa. El informe no es capaz de determinar con claridad cuáles fueron las informaciones que Kelly aportó a Gilligan. El informe asegura que Alastair Campbell no ordenó exagerar los informes, si bien manifestó el deseo del gobierno de que éstos fueran los más contundentes posibles. En opinión del Lord, tales presiones pudieron influir en el subconsciente de los redactores del informe. Lord Hutton estima que Hoon no es responsable de que el nombre de David Kelly saltara a la luz pública, aunque debería haber informado al científico que su nombre saldría.

El ataque más duro es la conclusión de que las informaciones de Gilligan fueron un ataque grave e infundado al gobierno. Fallaron los mecanismos de control editorial y seguimiento de las informaciones no contrastadas: el asunto de las armas de destrucción masiva deberían haberse investigado con mayor rigor. Tales acusaciones apuntaban directamente a Greg Dyke, director general, y al periodista Andrew Gilligan.

Las reacciones no se hicieron esperar. La BBC pide disculpas públicas y tres personas abandonan su cargo:

\footnotetext{
1 "La hora más triste de la BBC", La Vanguardia, 29 de enero de 2004.
} 
- El periodista Andrew Gilligan es apartado del programa estrella "Today"

- Gavyn Davies, presidente de la BBC, dimite

- Greg Dyke, director general de la BBC, dimite

Gavyn Davies dimite por las críticas vertidas en el informe Hutton a la Corporación, sobre todo en relación con las deficiencias del modelo editorial. El Consejo de Gobernadores está acusado de no tomar en consideración las reclamaciones y no investigar si los informes originales y las fuentes era exactas.

Lord Hutton acusa a la BBC de haber emitido dos noticias cuyas bases eran infundadas. Davies se defendió argumentando que no había ninguna motivación maligna y que la BBC no había informado mal deliberadamente. Al tiempo, el presidente cuestionaba alguna de las conclusiones del informe, ya que "si uno no puede escoger a quién le defienda ni presentar alegaciones". Además, la cuestión de las fuentes era un tema muy delicado, porque podría constituir una "amenaza a la libertad de prensa en este país". El periódico The Independent apoyaba la hipótesis de Davies y sostenía que Lord Hutton había juzgado con un doble rasero al gobierno y a la BBC, otorgando al primero el beneficio de la duda y atacando a la segunda en los mismos casos. Davies fue apoyado por Tessa Jowell, secretaria de Estado de Cultura, que aseguró que el presidente había hecho lo correcto.

Greg Dyke se disculpó "sin reservas" por aquellas cuestiones que se habían hecho mal durante las informaciones elaboradas por Andrew Gilligan y apuntó que la Corporación cambiaría alguno de sus procedimientos. Hutton insistía en su informe que los gobernadores de la BBC debía haber atendido a las reclamaciones presentadas por el gobierno, lo cual no era incompatible con la independencia editorial.

Andrew Marr, editor de política en los informativos de la BBC, señalaba que el informe no era más que un juicio elaborado por Lord Hutton, que había caído del lado de la versión política y oficial. Tony Blair sale airoso de la crisis, pero con las cuotas de popularidad más bajas de su mandato. Las encuestas publicadas por The Guardian revelan que el $48 \%$ de los votantes considera que Blair estaba mintiendo cuando decía que no autorizó que se hiciera público el nombre de David Kelly y que el $63 \%$ consideraba que debía dimitir si el informe le acusaba de mentir. No sucedió tal cosa, pero el informe sí que recalcaba que el gobierno había actuado de manera deshonorable, ilícita o engañosa por convertir el nombre del doctor Kelly en el campo de batalla con la BBC. Asimismo, apunta directamente al ministro Hoon, a quien acusa de fallar al señalar sin matices al doctor Kelly.

En conclusión, la mayoría de los británicos consideran que el gobierno manipuló los informes sobre las armas químicas y mintió deliberamente, si bien Lord Hutton no se pronuncia sobre la veracidad de estos informes. Su razonamiento consiste en que la advertencia de que Iraq podía hacer uso de armas de destrucción masiva en menos de 45 minutos estaba justificada, al basarse en una fuente que en aquellas circunstancias era considerada fiable. 


\subsection{La nueva dirección general}

El 5 de abril de 2004 el comité de selección de la BBC ha elegido a Michael Grade como presidente. Tony Blair se ha limitado a dar su plácet y firmar el nombramiento, si bien no era su candidato predilecto. Mark Byford ha ejercido el cargo de director general en funciones desde la dimisión de Greg Dyke en enero. El 28 de mayo de 2004 Mark Thompson, hasta entonces director ejecutivo de Channel 4, es nombrado director general de la BBC en sustitución de Greg Dyke, dimitido tras la publicación del informe del juez Hutton sobre el "caso Kelly". Toma posesión del cargo el 22 de junio y promete un cambio rápido y radical en la Corporación. Ha criticado al director general saliente porque la BBC "casi ha olvidado el mundo de fuera, una organización cuyo tono de vez podría a veces sonar puntiagudo y defensivo, arrogante incluso" ${ }^{2}$.

El primer punto que Thompson quiere modificar es la estructura de la organización, que considera amorfa. Thompson ha criticado la separación existente entre las divisiones de Production y Broadcasting, instaurada por John Birt y mantenida por Greg Dyke, porque no ha resultado exitosa para los objetivos de la Corporación.

Tiene previsto crear tres órganos que supervisen respectivamente las actividades comerciales, la dirección de creatividad (contenidos) y el periodismo realizado por la Corporación y reducir el comité ejecutivo. Respecto a las actividades comerciales, Thompson ha sugerido que hay que encontrar nuevas fuentes de ingresos: "Cuando vuelvo a la BBC, mirándola con nuevos ojos, lo que veo es que la mayoría de lo que realizamos tiene potencial creativo y económico. Pero también veo la necesidad de clarificar la estrategia y simplificar la complejidad". Asimismo, se tiene que revisar la cadena de valor de las actividades comerciales, ya que "BBC Worldwide y BBC Ventures están llenas de talento y han construido ya de hecho una enorme cantidad de valor para el público".

Para la dirección de creatividad, Thompson ha nombrado a Alan Yentob, a quien ha encargado que conecte con las comunidades de creadores ajenos a la BBC. El director considera que "sin grandes programas, grandes contenidos, no somos nadie. Sin embargo, las cuestiones sobre la creatividad pueden parecer lejos de los más alto de la BBC, siempre centrado en los debates acerca del dinero, la tecnología y la estrategia a largo plazo".

El nuevo departamento de periodismo tiene que asegurar que la información es transparente y equilibrada: "Deseo que no sólo los directivos, sino los periodistas y los editores trabajen de la mano para crear una cultura que combine los más altos modelos con el espíritu de la empresa periodística e independiente". Bajo Dyke, contaba con 17 miembros, lo que Thompson considera "demasiado grande y amorfo para ser realmente efectivo". El nuevo comité contará sólo con nueve miembros, ya que "era a menudo un buen lugar para debatir los temas de la Corporación, pero las decisiones rara vez eran tomadas por el comité. No jugó ningún papel desta-

\footnotetext{
2 "Radical shakeup starts at BBC", The Guardian, 23 de junio de 2004.
} 
cado en el asunto Hutton, a pesar de que reunía mucha experiencia” ${ }^{3}$. En relación con la situación financiera, Thompson considera que es demasiado tirante, por lo que se recortarán muchos gastos para cuadrar las cuentas.

La respuesta de la Corporación al informe de Lord Hutton ha sido la publicación del documento The Neil Report. Statement by the Board of Governors. El informe ha sido dirigido por Ron Neil, antiguo director de informativos de la BBC, con el objeto de ser asumido e implementado por completo. El informe se ha publicado el 23 de junio de 2004.

Las principales aportaciones giran en torno al cuidado de la información y la redacción de las noticias ${ }^{4}$. Textualmente se dice que la exactitud y la precisión son la cumbre ("paramount") del periodismo de la BBC. Tiene que basarse en la evidencia fuerte y ser reforzada por la exactitud de las notas, que es una habilidad periodística fundamental y que debería formar parte de la formación periodística de la BBC. Al tiempo, las argumentaciones serias y potencialmente difamatorias tienen que ser tenidas en consideración antes de la emisión de una noticia. Para ello, los editores y los productores ejecutivos tienen que velar para que los contenidos respeten los valores editoriales de la BBC.

Los presentadores tienen que responder ante su editor por el respeto de los valores centrales de la línea editorial de la BBC. Según el informe, los valores centrales que rigen la Corporación son los siguientes:

- La verdad y la exactitud: el periodismo de la BBC debe guiarse por la mayor precisión y estar bien fundamentado en evidencias visibles

- Servir al interés público: se deben priorizar las noticias significantes para la audiencia y ser claro cuando se cuestionen las figuras públicas

- La imparcialidad y la diversidad de opiniones: la información debe primar sobre la opinión y se debe argumentar la evidencia con una amplia gama de opiniones

- La independencia

- La responsabilidad: la BBC debe mantener la confianza de la audiencia y estar abierta a cometer errores, porque se puede y debe disculpar, así como aprender de ellos

En relación con las fuentes, el informe asegura que su protección y confidencialidad es un principio fundamental del periodismo, pero no se puede garantizar el anonimato automáticamente. Si la fuente tiene que ser anónima, la BBC tiene que explicar por qué lo es y por qué tiene la confianza de la BBC. Las quejas y reclamaciones necesitan un nuevo método, ya que los mecanismos establecidos no han funcionado durante la crisis del doctor Kelly. Finalmente, la formación

\footnotetext{
3 "New BBC boss's key plans", The Guardian, 30 de junio de 2004.

${ }^{4}$ BBC: The Neil Report. Statement by the Board of Governors. BBC. Londres, 2004.
} 
continua en todos los niveles es vital en un mundo que cambia muy rápido. Recomendamos que la BBC establezca un college de periodismo bajo el liderazgo de un decano académico.

De todos los aspectos del informe, la formación continua de los periodistas es el aspecto que más interesa para el reto digital. La BBC ha decidido recuperar la credibilidad, que es la base principal de la marca BBC. La inversión en formación es el primer paso para recuperar esta credibilidad. Por otro lado, el informe avala la decisión del equipo gestor dirigido por Greg Dyke de prohibir a los periodistas de la BBC mantener columnas de opinión en otros medios de comunicación, aunque no sean competencia directa, ya que ponen en peligro la independencia de la Corporación.

Desde un punto de vista crítico, el informe no presenta una autocrítica de la actuación que llevó a la corporación al enfrentamiento con el gobierno, aunque se sugieran algunas mejoras en el funcionamiento interno para que no pueda volver a cuestionarse a la profesionalidad de los informativos de la Corporación.

\section{LA REVISIÓN DEL SERVICIO PÚBLICO AUDIOVISUAL}

El 6 de noviembre de 2004 la Ofcom ha comenzado formalmente la revisión del servicio público audiovisual, proceso que durará más de un año y que afectará a los operadores que cumplen alguna misión de servicio público. El resultado de la investigación servirá de base para la revisión gubernamental de la Royal Charter, que tendrá lugar en 2006. El equipo propuesto por la Ofcom está compuesto por Ed Richards, como máximo responsable, Robin Foster, responsable de estrategias, y Tim Suter, responsable de contenidos. Su propuesta de trabajo está publicada por la Ofcom bajo el título Is television special?.

La Communications Act 2003 insta a la Ofcom a que defina los propósitos y principios del servicio público audiovisual, que incluye los contenidos y los servicios de la BBC, Channel 3, Channel 4, Channel 5 y S4C. De partida, la clause 264 de la Communications Act 2003 plantea el marco legal de cuáles son los propósitos del servicio público de televisión:

- El ser compartida por un amplio rango de sujetos

- La atención a la más amplia variedad de audiencias en diferentes horarios y mediante diferentes tipos de contenidos

- El mantenimiento de altos patrones de producción

El cumplimiento de tales propósitos supone informar, entretener y educar a la sociedad, así como el apoyo a la diversidad audiovisual de modo que la compra de producciones ajenas se diversifique lo más posible. Los contenidos a considerar para cumplir con objetivos incluyen programas que muestren la actividad cultural del país, servicios informativos, deporte y entretenimiento, educación, ciencia, religión, programas de contenido social, contenidos para los menores y programas para las diferentes comunidades que conviven en Reino Unido. 
La revisión incluirá los servicios ofrecidos por los nuevos medios, dada su relación manifiesta con la televisión, así como otros medios por el estilo. Al tiempo, la Ofcom ha planteado la revisión estratégica del sector de las telecomunicaciones. Finalmente, la radio no se incluirá en este informe.

La revisión consta de tres fases. El marco de actuación propuesto es el resultado de las tres etapas que veremos a continuación y que se resumen en el siguiente esquema.

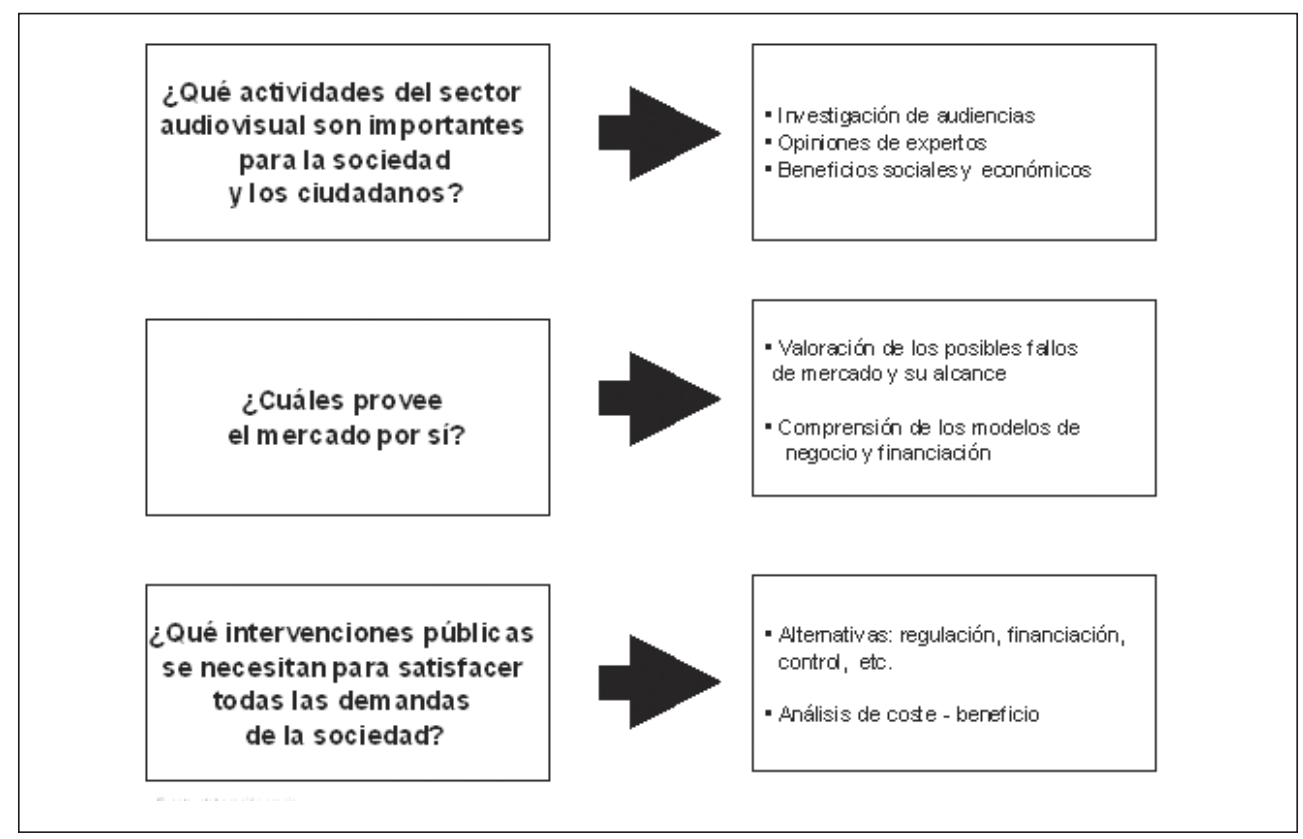

Ilustración 1

La primera etapa es un análisis de la situación actual y se desarrollará desde octubre de 2003 hasta la primavera de 2004. Los objetivos declarados son los siguientes:

- La valoración detallada de la extensión, tomados en su conjunto, del servicio público audiovisual que proveen las citadas cadenas

- El establecimiento de los propósitos y las definiciones del servicio público audiovisual para los individuos, las familias, los ciudadanos y para Reino Unido, así como conocer la valoración de la opinión pública sobre cómo se están cumpliendo tales propósitos

- La valoración de los costes de provisión del servicio público audiovisual, la financiación disponible y los posibles beneficios derivados del mismo 
El método a desarrollar para la investigación consiste en un análisis de aquello que la audiencia considera los principales propósitos y objetivos. La opinión pública se ha obtenido del análisis cualitativo sobre las preferencias y actitudes de 6.000 espectadores británicos. La investigación incluye un estudio avanzado y examina las actitudes y las necesidades de los grupos sociales y étnicos específicos en relación con las demandas propias en ámbitos como la educación, la cultura y la ciudadanía.

Los primeros resultados de la investigación son los siguientes ${ }^{5}$ :

- El principal propósito de la televisión es el entretenimiento

- La televisión tiene una responsabilidad destacada para con la sociedad

- Este modo de comprender la televisión es compartido por la mayoría de los grupos demográficos

- El servicio público de televisión se compone de muchos elementos

- Mientras que muchos géneros son importantes para la sociedad, existe cierta insatisfacción con la provisión actual de contenidos

- La mayoría valora como pobre la innovación en la programación

- La programación apropiada es un elemento clave

- Los telespectadores valoran positivamente la actual estrategia de provisión de señal terrestre

- La televisión podría tener un rol más proactivo

- Los telespectadores desean mantener la provisión de los servicios públicos audiovisuales

El estudio refleja la manera en que los telespectadores ven la televisión y valoran los contenidos emitidos por las emisoras por vía terrestre. Brian McNair comparte este modelo de revisión, frente al sistema tradicional que ha sostenido la BBC desde su origen:

Esta vuelta de tuerca requiere repensar el significado de los términos clave como "público" o "servicio", expandiendo el rango de aquello que puede ser incluido dentro de éstos. Se requiere una ruptura con el pasado paternalista, abandonando la idea de que el rol de la cultura es iluminar e instruir a las masas ineducadas, llenándolas mediante instituciones como la $B B C^{6}$.

${ }^{5}$ Cfr. OFCOM: The role of society: audience opinions and perceptions. Ofcom. Londres, 2004.

${ }^{6}$ MCNAIR, Brian: "British public service broadcasting beyond 2006: which publics, what services?". Paper presentado en la conferencia RIPE 2004: Mission, Management \& Markets: Public Service Broadcasting and the Cultural Commons. 
Frente al modelo de posguerra, McNair reclama que el futuro del servicio público audiovisual descansa no en el discurso paternalista sobre qué es bueno para la audiencia en términos culturales, sino en el reconocimiento por parte de las televisiones de la validez de los gustos populares. En suma, los servicios públicos audiovisuales tienen que reunir la satisfacción de los gustos populares en un entorno competitivo y la provisión de recursos culturales necesarios para la ciudadanía.

La segunda etapa se realiza durante la primavera y el verano de 2004. Los objetivos marcados son los siguientes:

- La valoración de las prospectivas de los servicios públicos de televisión dentro de los cambios del mercado, incluyendo los desarrollos del mercado (tecnologías, audiencias, nuevos servicios, etc.), el camino hacia la digitalización, la emergencia de nuevos modelos de negocio y el futuro procedimiento financiero del sector.

- La valoración de la economía de la televisión actual y de futuro. Incluye una valoración de las opciones de financiación y del impacto de la provisión de los servicios públicos audiovisuales en el sector.

- La revisión preliminar de las opciones disponibles para mantener y reforzar la calidad de los servicios públicos audiovisuales.

Para conseguir los objetivos, se realizará un análisis de las tendencias del mercado en campos como la industria, la audiencia y la tecnología durante los cinco o diez años. Asimismo, se investigará el último quinquenio de los operadores, quienes proveerán los datos necesarios sobre los costes, los ingresos y el volumen de programación, así como los gastos asociados a cada canal y cómo han contribuido a la consecución de los objetivos de servicio público.

La opinión del partido conservador queda reflejada en el informe Beyond Charter Renewal, publicado en febrero de 2004. El documento defiende la abolición del canon y la privatización de la mayor parte de las funciones del servicio público audiovisual que ejerce la BBC.

Por último, la tercera fase se desarrolla durante el verano y el otoño de 2004. Como resultado se publicará un informe final, cuyos objetivos son:

- Una valoración comprensiva de la extensión y el modo en que se está desarrollando el servicio público de televisión.

- Los conceptos clave y los desafíos para el mantenimiento o el refuerzo de la provisión del servicio público de televisión para el futuro.

- Un conjunto de opciones para asegurar la futura provisión de servicio público de televisión.

El objetivo final de la revisión de la Ofcom es acordar un marco de actuación que cimente las políticas y los servicios públicos para el audiovisual para el próximo 
decenio: "Cualquiera sea el punto de partida adoptado, las conclusiones tienen que ser las mismas: es importante desarrollar algunas políticas regulatorias de consenso pensando tanto en el largo plazo de los ingresos del audiovisual (esto al final será un elemento de juicio, conformado por un pensamiento lúcido sobre el tipo de sociedad en que queremos vivir) y la comprensión de qué podría proveer un sistema basado en el mercado (dados unos mercados que son usualmente mejores en la redistribución de los recursos y satifaciendo las necesidades de los consumidores que los sistemas de ordeno y mando, incluso aquellos que están en sintonía con el servicio público audiovisual)" ".

Con la idea de desarrollar unas categorías válidas para el modelo británico se ha propuesto un esquema específico de valoración de los diferentes objetivos del servicio público, porque "para cada objetivo es posible desarrollar unas medidas que puedan ser juzgadas a lo largo del tiempo, ver cómo se están cumpliendo los contenidos (de las medidas) y permitir la comparación entre emisoras",

El esquema propuesto incluye los siguientes puntos ${ }^{9}$ :

Tabla 1

\begin{tabular}{|c|c|}
\hline $\begin{array}{l}\text { Variedad } \\
\text { y } \\
\text { equilibrio } \\
\text { de contenidos }\end{array}$ & $\begin{array}{l}\text { - Diversidad de géneros } \\
\text { - Amplitud y variedad de géneros, subgéneros y formatos } \\
\text { - Inversión comparada entre géneros } \\
\text { - Disponibilidad de contenidos en horas de máxima audiencia }\end{array}$ \\
\hline Diversidad & $\begin{array}{l}\text { - Programación que cumpla las necesidades y las demandas de las } \\
\text { diferentes comunidades } \\
\text { - Programación para distintos tipos de targets } \\
\text { - Representación de los distintos puntos de vista } \\
\text { - Programación demandada a distintas productoras } \\
\text { - Promoción de la representación de las distintas comunidades }\end{array}$ \\
\hline Calidad & $\begin{array}{l}\text { - Originalidad e innovación } \\
\text { - Cumplimiento con los cánones de decencia y el buen gusto } \\
\text { - Producción de alta gama } \\
\text { - Contenidos ambiciosos: desafiantes, provocadores y estimuladores de } \\
\text { la audiencia }\end{array}$ \\
\hline $\begin{array}{l}\text { Valores } \\
\text { sociales }\end{array}$ & $\begin{array}{l}\text { - } \text { Identidad cultural: } \\
\text { o Reflejo de Reino Unido, sus naciones y regiones } \\
\text { o Promoción de las artes y el patrimonio nacional } \\
\text { - La democracia informada: } \\
\text { o Disponibilidad e imparcialidad de los informativos } \\
\text { o Promoción de las campañas de acción social } \\
\text { - La ciudadanía educada: } \\
\text { o Contenidos y programación específica para la educación } \\
\text { o Programación específica para la infancia } \\
\text { o } \text { Variedad en los servicios informativos }\end{array}$ \\
\hline
\end{tabular}

${ }^{7}$ FOSTER, Robin; EGAN, Jim y SIMON, Jonathan: Measuring Public Service Broadcasting. Ofcom. Londres, 2003. P. 5.

${ }^{8}$ Ibíd., p.10.
${ }_{9}^{9}$ Ibíd., p.10. 
Las tres fases tratan de dar respuesta a las demandas de la sociedad británica, en el entorno de la transformación del estado del bienestar a la luz de la sociedad informacional. El examen trata de medir tanto cualitativa como cuantitativamente el servicio público audiovisual y como hemos visto es posible establecer unas categorías que permitan la evaluación a largo plazo; al tiempo se impone un marco de jerarquización de las obligaciones del servicio público audiovisual. Igualmente se valora el grado de cumplimiento por parte de las televisiones.

Finalmente, después del informe de Lord Hutton se han oído voces críticas con la BBC, porque puede convertirse en un medio "suave" con el poder ante la posible reprimenda pública posterior. En el caso de David Kelly, la información era correcta y cumplió con su función de servicio público. Sin embargo, los altos cargos han tenido que dimitir por el escándalo. Por tanto, la revisión de la BBC tiene que ser absoluta: desde la financiación a la misión, pasando por el nombramiento de altos cargos y la presentación de reclamaciones. El informe Hutton ha sido tenido en cuenta a la hora de comenzar la revisión de la Royal Charter. Tessa Jowell, secretaria de Estado de Cultura, opinaba que el informe haría de la BBC una corporación más fuerte y más independiente del gobierno.

\section{CONCLUSIONES}

El audiovisual, como todas las políticas públicas de la comunicación, es un tema de debate constante en Reino Unido. A lo largo de su historia, el poder ejecutivo ha nombrado una y otra vez comités y comisiones para estudiar la situación presente y futura del sector. La BBC siempre ha tenido un cuerpo de thinks tanks que ha opinado sobre su futuro a corto y medio plazo, por lo que el informe de Lord Hutton no es una novedad radical. Siguiendo las recomendaciones, la Ofcom ha abierto un proceso que durará más de un año y que afecta a todos los operadores que cumplen alguna función de servicio público. El resultado de la investigación servirá de base para la revisión gubernamental de la Royal Charter, que tendrá lugar en 2006. El examen trata de medir tanto cualitativa como cuantitativamente el servicio público audiovisual mediante categorías conceptuales y valorativas que permitan la evaluación a largo plazo, al tiempo se establece un marco de comparación y jerarquización de obligaciones de servicio público, así como el grado de cumplimiento por parte de las televisiones.

- Durante 2003 y 2004, la BBC ha tenido que hacer frente a una crisis estructural, que ha incidido en la pérdida de audiencia y de credibilidad. Sin embargo, BBC ha salido fortalecida, porque ha aprendido la lección, ha limpiado las heridas y se adentrado en una nueva etapa. La Corporación sufrió un cataclismo tras la publicación de las conclusiones del informe de Lord Hutton sobre el caso Kelly. El presidente de la Corporación y el Director General dimitieron, a pesar de que existen sombras acerca de su implicación directa en el caso. Recordemos, además, que el caso estalló por el comen- 
tario un periodista en un programa de radio matinal, que no era el programa estrella y, muchos menos, el director de los servicios informativos.

- Para aumentar la calidad de los servicios informativos, la BBC encargó un informe para aprovechar las fortalezas y disminuir las debilidades. El informe, conocido como Neil Report, ha decidido recuperar la credibilidad, que es la base principal de la marca BBC. Con tal objetivo, se ha preparado un proceso de formación continua de los periodistas. La inversión en formación se justifica porque aumenta el valor añadido e incrementa el espacio de credibilidad. Por otro lado, el informe avala la decisión del equipo gestor dirigido por Greg Dyke de prohibir a los periodistas de la BBC mantener columnas de opinión en otros medios de comunicación, aunque no sean competencia directa, ya que ponen en peligro la independencia de la Corporación.

\section{BIBLIOGRAFÍA CITADA}

BBC: The Neil Report. Statement by the Board of Governors. BBC. Londres, 2004.

FOSTER, Robin; EGAN, Jim y SIMON, Jonathan: Measuring Public Service Broadcasting. Ofcom. Londres, 2003.

MCNAIR, Brian: "British public service broadcasting beyond 2006: which publics, what services?". Paper presentado en la conferencia RIPE 2004: Mission, Management \& Markets: Public Service Broadcasting and the Cultural Commons.

OFCOM: The role of society: audience opinions and perceptions. Ofcom. Londres, 2004.

(Recibido el 8-11-05, aceptado el 15-03-06) 